\title{
Comparison of the cellular transport mechanism of cationic, star-shaped polymers and liposomes in $\mathrm{HaCat}$ cells
}

Heng-Cong Luo, ${ }^{1,2, *} \mathrm{Na}$ Li, ',* Li Yan,' Kai-jin Mai, ${ }^{3}$ Kan Sun, ' Wei Wang,' GuoJuan Lao,' Chuan Yang,' Li-Ming Zhang, ${ }^{3}$ Meng Ren'

'Department of Endocrinology, Sun Yat-Sen Memorial Hospital, Guangdong Provincial Key Laboratory of Malignant Tumor Epigenetics and Gene Regulation Medical Research Center, Sun Yat-Sen University, Guangzhou, People's Republic of China; ${ }^{2}$ Department of Endocrinology, The Third Affiliated Hospital of Guangzhou Medical University, Guangzhou, People's Republic of China; ${ }^{3} \mathrm{School}$ of Materials Science and Engineering, School of Chemistry, Sun Yat-Sen University, Guangzhou, People's Republic of China

*These authors contributed equally to this work

Correspondence: Meng Ren

Department of Endocrinology, Sun

Yat-Sen Memorial Hospital, Guangdong

Provincial Key Laboratory of Malignant

Tumor Epigenetics and Gene Regulation Medical Research Center, No 107 Yan Jiang West Road, Sun Yat-Sen University, Guangzhou 510120, People's Republic of

China

Tel +86208I332286

Fax +86 2081332404

Email renmeng80@।39.com

Li-Ming Zhang

School of Materials Science and Engineering, School of Chemistry, Sun Yat-Sen University, No I35, Xingang West Road, Guangzhou 520175, People's Republic of China

Tel +8620 84I I 2354

Email ceszhlm@mail.sysu.edu.cn
This article was published in the following Dove Press journal:

International Journal of Nanomedicine

7 February 2017

Number of times this article has been viewed

Abstract: Several biological barriers must be overcome to achieve efficient nonviral gene delivery. These barriers include target cell uptake, lysosomal degradation, and dissociation from the carrier. In this study, we compared the differences in the uptake mechanism of cationic, star-shaped polymer/MMP-9siRNA complexes ( $\beta$-CD-(D3)7/MMP-9siRNA complexes: polyplexes) and commercial liposome/MMP-9siRNA complexes (Lipofectamine ${ }^{\circledR} 2000 /$ MMP-9siRNA complexes: liposomes). The uptake pathway and transfection efficiency of the polyplexes and liposomes were determined by fluorescence microscopy, flow cytometry, and reverse transcriptase-polymerase chain reaction. The occurrence of intracellular processing was assessed by confocal laser scanning microscopy. Endosomal acidification inhibitors were used to explore the endosomal escape mechanisms of the polyplexes and lysosomes. We concluded that the polyplexes were internalized by non-caveolae- and non-clathrin-mediated pathways, with no lysosomal trafficking, thereby inducing successful transfection, while the majority of liposomes were internalized by clathrin-dependent endocytosis (CDE), caveolae-mediated endocytosis, and macropinocytosis, and only CDE induced successful transfection. Liposomes might escape more quickly than polyplexes, and the digestion effect of acidic organelles on liposomes was faint compared to the polyplexes, although both complexes escaped from endolysosomes via the proton sponge mechanism. This may be the key aspect that leads to the lower transfection efficiency of the $\beta$-CD-(D3)7/MMP-9siRNA complexes. The present study may offer some insights for the rational design of novel delivery systems with increased transfection efficiency but decreased toxicity.

Keywords: $\beta$-CD-(D3)7, liposome, endocytosis, endolysosomal escape, intracellular trafficking

\section{Introduction}

Diabetic foot ulcers are a complication of chronic diabetes and an important factor of morbidity and mortality in patients with diabetes. Our previous study showed that the application of small interfering RNA (siRNA) to inhibit matrix metalloproteinase (MMP-9) expression could promote the process of cutaneous wound healing. ${ }^{1}$ However, as siRNA is large and charged, it has difficulty in traversing the plasma membrane on its own. Thus, an appropriate gene delivery system is required for its efficient cellular uptake. ${ }^{2}$

In our previous study, we synthesized a cationic, star-shaped polymer consisting of a $\beta$-CD core and poly(amidoamine) dendron arms, ie, $\beta$-CD-(D3)7. The ability of $\beta$-CD-(D3)7 to condense MMP-9siRNA into particulate structures was confirmed by agarose gel electrophoresis, particle size measurements, and transmission electron microscopy observations. ${ }^{1}$ However, this previous study showed that the submit your manuscript

Dovepress $f y$ in 0 cC. hereby accept the Terms. Non-commercial uses of the work are permitted without any further permission from Dove Medical Press Limited, provided the work is properly attributed. For permission for commercial use of this work, please see paragraphs 4.2 and 5 of our Terms (https://www.dovepress.com/terms.php). 
$\beta$-CD-(D3)7/MMP-9siRNA complexes (also denoted as polyplexes) had a higher internalization rate but a lower interference effect compared with commercial liposome gene vector-Lipofectamine ${ }^{\circledR} 2000$ (Thermo Fisher Scientific, Waltham, MA, USA)/MMP-9siRNA complexes (also denoted as lip2000/MMP-9siRNA complexes or liposomes; uptake rates of $98.4 \%$ vs $28.2 \%$ and interference rates of $44.2 \%$ vs $77.2 \%$ ). We speculated that these results may be related to the extracellular and intracellular barriers. Past research has shown that to achieve successful transfection, nonviral delivery systems, such as those delivering siRNA, need to overcome several cellular barriers, including being internalized by cells, escaping from endolysosomes, and dissociating the cargo from the complex for delivery into the cytoplasm. ${ }^{3}$

Endocytosis has been established as the main mechanism for the uptake of nonviral vectors into the cells. ${ }^{4}$ Endocytosis can be classified into five categories: phagocytosis, clathrin-dependent endocytosis (CDE), caveolae-mediated endocytosis (Cav-ME), non-clathrin- and non-caveolae dependent endocytosis, and macropinocytosis. Phagocytosis is typically restricted to specialized mammalian cells. CDE pathway is characterized by the formation of endosomes through the constriction and fission of clathrin-coated pits triggered by the GTPase dynamin. In Cav-ME pathway, the composition of the primary endocytic vesicle was in relation to lipid rafts. Lipid rafts are freely floating plasma membrane microdomains that are comprised of a combination of glycosphingolipids and protein receptors where cellular processes are compartmentalized. On the contrary, vesicles formed during macropinocytosis, which result from actindriven membrane protrusions that collapse onto the engulfed nanoparticle and fuse with the plasma membrane, can contain lipid rafts together with nonraft membrane domains. Non-clathrin- and non-caveolae-dependent endocytosis has not been thoroughly studied, but may involve the adenosine diphosphate-ribosylation factor 6 (ARF6)-dependent route and the RhoA-dependent pathway, among others. ${ }^{5}$

In our study, we compared $\beta$-CD-(D3)7/MMP-9siRNA complexes with lip2000/MMP-9siRNA complexes using endocytosis inhibitors, endosomal acidification inhibitors, and organelle fluorescent probes in terms of the following elements: cellular uptake, intracellular trafficking and transfection efficiency after internalization by different uptake modes, and potential endolysosomal escape mechanisms. The aim of this work is to provide some insights for further improving the molecular design and thereby the transfection efficiency of the polycationic amphiphilic cyclodextrin vector.

\section{Materials and methods Materials}

The $\beta$-CD-(D3)7 synthesis and $\beta$-CD-(D3)7/MMP-9siRNA complexes preparation methods were reported in our previous publication. ${ }^{1}$ Figure 1 shows the chemical structure of $\beta$-CD-(D3)7. Lipofectamine ${ }^{\circledR} 2000$ was purchased from Thermo Fisher Scientific. Human keratinocyte HaCat cells were purchased from the Chinese Academy of Sciences Cell Bank (Shanghai, People's Republic of China). $\alpha$-Modified Eagle Medium ( $\alpha$-MEM) and fetal bovine serum (FBS) were purchased from GIBCO (Grand Island, NY, USA). Cell Counting Kit-8 (CCK-8) was purchased from Dojindo (Kumamoto, Japan). Endocytosis inhibitors, such as M- $\beta-C D$, genistein, chlorpromazine, and amiloride and the endosomal acidification inhibitors, bafilomycin A1 and chloroquine, were purchased from Sigma (Schnelldorf, Germany). The roles of the inhibitors are shown in Table 1. The fluorescent nuclear probe Hoechst 33342 and the fluorescent lysosomal probe LysoTracker DND-99 were purchased from Thermo Fisher Scientifice. The fluorescently labeled organelle fluorescent probes used in the study are shown in Table 2. All other reagents were of analytical grade and used without further purification. MMP-9 siRNA was purchased from GenePharma (Suzhou, People's Republic of China). The sequences of the human MMP-9siRNA were as follows: sense, 5'-CUAUGGUCCUCGCCUGAATT-3'; antisense, 5'-UUCA GGGCGAGGACCAUAGTT-3'.

\section{Cell culture}

HaCat cells were cultured in $\alpha$-MEM supplemented with $10 \%$ FBS, penicillin $(100 \mathrm{U} / \mathrm{mL})$ and streptomycin $(100 \mathrm{mg} / \mathrm{mL})$ (Beyotime, Haimen, People's Republic of China) at $37^{\circ} \mathrm{C}$ in a humid atmosphere with $5 \% \mathrm{CO}_{2}$.

\section{Treatment with endocytosis inhibitors}

Effects of different endocytosis inhibitor concentrations on $\mathrm{HaCat}$ cell activity

The following inhibitors were used: $3-8 \mathrm{mM}$ M- $\beta-\mathrm{CD}$ (Sigma Cat No: C4555), 100-400 $\mu \mathrm{M}$ genistein (Sigma Cat No: 91955), 5-10 $\mu \mathrm{g} / \mathrm{mL}$ chlorpromazine (Sigma Cat No: C8138), and 25-75 $\mu \mathrm{M}$ amiloride (Sigma Cat No: A7410). Cells were treated with endocytosis inhibitors for $1 \mathrm{~h}$ at $37^{\circ} \mathrm{C}$. Subsequently, polyplexes or liposomes were added and the cells were incubated for another $4 \mathrm{~h}$. Then, $10 \mu \mathrm{L}$ of CCK-8 reagent was added to the plate and incubated for another $3 \mathrm{~h}$. After incubation, the optical density (OD) was measured at 450 and $630 \mathrm{~nm}$ using a plate spectrophotometer. Each group had six parallel replicates. Cell viability was calculated as 


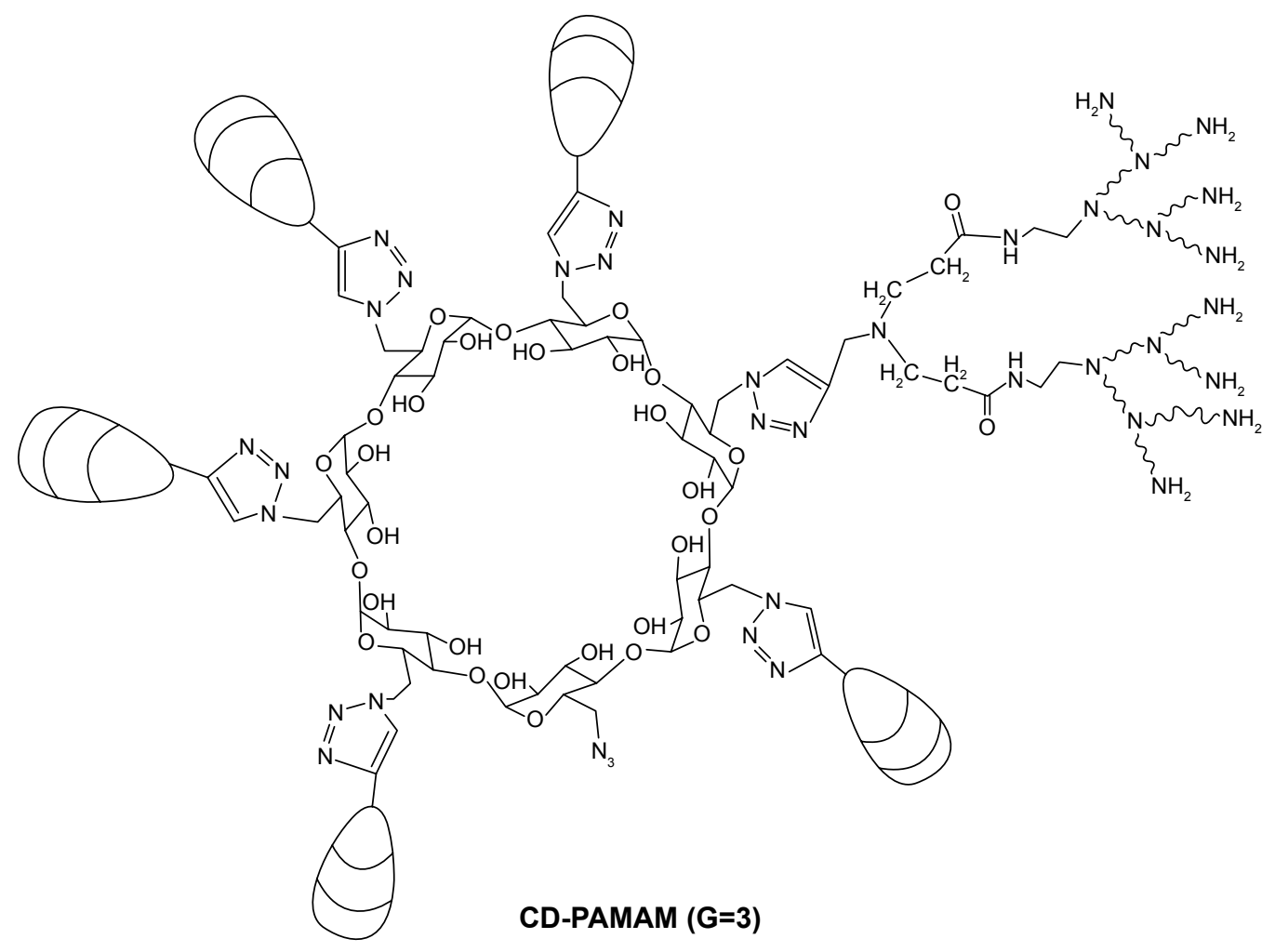

Figure I Chemical structure of $\beta-C D-(D 3) 7$.

follows: cell viability $(\%)=(\mathrm{OD}[$ sample $]-\mathrm{OD}[$ blank $] / O D$ [control] - OD [blank]) $\times 100$. As a positive control, cells were treated with $\beta$-CD-(D3)7/MMP-9siRNA complexes or lip2000/MMP-9siRNA complexes in the absence of any inhibitors. Three independent experiments were performed using HaCat cells. The most efficient concentration of each endocytosis inhibitor with the lowest cytotoxicity was selected as described later.

\section{Effects of different endocytosis inhibitors on complex uptake efficiency}

$\mathrm{HaCat}$ cells were seeded $24 \mathrm{~h}$ prior to transfection in 24-well plates $(10,000$ cells/well). Cells were treated with chlorpromazine $(8 \mu \mathrm{g} / \mathrm{mL})$, genistein $(100 \mu \mathrm{M})$, amiloride $(50 \mu \mathrm{M})$, or M- $\beta$-CD ( $3 \mathrm{mM})$ in Opti-MEM medium for $1 \mathrm{~h}$ at $37^{\circ} \mathrm{C}$ before transfection. Subsequently, polyplexes or liposomes were added and the cells were incubated for an additional $4 \mathrm{~h}$. The siRNA was labeled with 5-carboxyfluorescein (5-FAM). The cells were viewed by fluorescence microscopy before being detached by trypsinization. After detachment and centrifugation, the cells were resuspended and analyzed using a FACSCalibur system (BD Biosciences, San Jose, CA, USA). Ten thousand cells were measured in each sample.

\section{Intracellular process of complex uptake by different} endocytosis pathways

Eight thousand HaCat cells were seeded in dishes and cultured for $24 \mathrm{~h}$. The cells were treated as described earlier. Then,

Table I Inhibitors and markers used for different endocytic pathways

\begin{tabular}{|c|c|c|}
\hline Pathway & Inhibitor & Mechanism \\
\hline Clathrin-mediated endocytosis (CDE) & Chlorpromazine & Interacts with clathrin, to block CDE pathway \\
\hline \multirow{2}{*}{$\begin{array}{l}\text { Caveolae-mediated endocytosis } \\
\text { (Cav-ME) }\end{array}$} & Genistein & A tyrosine-kinase inhibitor, to block Cav-ME pathway \\
\hline & Methyl- $\beta$-cyclodextrin $(M-\beta-C D)$ & $\begin{array}{l}\text { Inclusion complexes with cholesterol, simultaneous inhibition } \\
\text { of CDE and Cav-ME pathway }\end{array}$ \\
\hline Macropinocytosis & Amiloride & Blocks $\mathrm{Na}^{+} / \mathrm{H}^{+}$pump, to block macropinocytosis \\
\hline \multirow[t]{2}{*}{ Acidification of endosomes } & Chloroquine & Increases endosomal PH, inhibits the acidification of endosomes \\
\hline & Bafilomycin Al & Blocks $\left(\mathrm{H}^{+}\right)$ATPase, inhibits the acidification of endosomes \\
\hline
\end{tabular}


Table 2 Characteristics of the dyes used

\begin{tabular}{lllll}
\hline & Dye/marker & Application/purpose & Excitation $_{\max }(\mathbf{n m})$ & Emission $_{\max }(\mathbf{n m})$ \\
\hline Liposome marker & LysoTracker DND-99 & Incorporated into liposome membrane & 577 & 590 \\
Cell marker & Hoechst 33342 & Nucleolus & 346 & 460 \\
Polyplexes or liposomes marker & 5-FAM & Incorporated into MMP-9siRNA & 494 & 522 \\
\hline
\end{tabular}

the cells were extensively washed with phosphate-buffered saline (PBS) and treated with 0.04\% trypan blue to quench extracellular fluorescence. Subsequently, the cells were treated with $1 \mu \mathrm{M}$ LysoTracker DND-99 for $1 \mathrm{~h}$ at $37^{\circ} \mathrm{C}$ and the nuclei dyed with Hoechst $33342(1 \mu \mathrm{g} / \mathrm{mL})$ for $30 \mathrm{~min}$ at $37^{\circ} \mathrm{C}$. The samples were examined using laser scanning confocal microscopy (Zeiss AxioPlan 2; Carl Zeiss Meditec AG, Jena, Germany) as a preliminary investigation of the intracellular processing of polyplexes and liposomes internalized by different pathways.

Effect of different endocytosis inhibitors on complex transfection efficiency

After treatment with endocytosis inhibitors and polyplexes or liposomes, the cells were washed $3 \times$ with PBS, and then $\alpha$-MEM supplemented with $10 \%$ FBS was added and the cells further incubated for another $48 \mathrm{~h}$. As a positive control, cells were transfected with complexes without inhibitors. MMP-9 mRNA expression levels were detected to evaluate the effect of the endocytosis inhibitors on transfection efficiency. MMP-9 mRNA transcription levels were determined by quantitative reverse-transcription PCR (RT-qPCR; Takara, Beijing, People's Republic of China) according to the manufacturer's instructions. In brief, total RNA was isolated from cells using RNA Plus reagent (Takara). Then, $400 \mathrm{ng}$ of total RNA was used as the template for first-strand cDNA synthesis by a PrimeScript ${ }^{\circledR}$ RT Master Mix Perfect Real-Time system (Takara). In all, $2 \mu \mathrm{L}$ of each reverse-transcription reaction was amplified in a $20 \mu \mathrm{L}$ PCR system using ABI7500. The results were normalized by the threshold cycle (CT) of GAPDH.

\section{Imaging of intracellular fluorescently labeled polyplexes or liposomes at different times}

Cells were seeded in dishes $(8,000$ cells/dish) and cultured for $24 \mathrm{~h}$. Then, the cells were transfected with complexes for $2 \mathrm{~h}$, extracellular fluorescence was quenched with $0.04 \%$ trypan blue, and the cells were incubated with LysoTracker DND-99 for $1 \mathrm{~h}$. Subsequently, the cell nuclei were dyed with Hoechst 33342 for $30 \mathrm{~min}$; the cells were then rinsed with PBS and incubated with $\alpha$-MEM supplemented with $10 \%$ FBS for 0,8 , or $20 \mathrm{~h}$, respectively. Finally, the cells were fixed with 3\% paraformaldehyde and observed by confocal microscopy. Images of cells containing fluorescently labeled polyplexes or liposomes were obtained at 4,12 , and $24 \mathrm{~h}$ after transfection.

\section{Endosomal release of polyplexes or liposomes}

Cell viability after treatment with endosomal acidification inhibitors was assessed by CCK-8 assays, as described in the "Effects of different endocytosis inhibitor concentrations on HaCat cell activity" section. The following reagents were used: $50-150 \mu \mathrm{M}$ chloroquine and, $50-200 \mathrm{nM}$ bafilomycin A1. The concentrations of these reagents with the lowest toxicities were selected.

Ten thousand HaCat cells were seeded in 24 well plates for $24 \mathrm{~h}$ and then treated with chloroquine $(50 \mu \mathrm{M})$ or, bafilomycin $\mathrm{A} 1(50 \mathrm{nM})$ for $1 \mathrm{~h}$ at $37^{\circ} \mathrm{C}$ before transfection. Polyplexes or liposomes were then added and incubation continued for $4 \mathrm{~h}$. Subsequently, the cells were washed $3 \times$ with PBS, and $\alpha$-MEM supplemented with $10 \%$ FBS was added followed by an additional incubation of $48 \mathrm{~h}$. Complexes not treated with inhibitors were used as positive controls. MMP-9 mRNA transcription levels were determined by RT-qPCR.

\section{Statistical analysis}

Quantitative data are expressed as the mean \pm standard deviation. Statistical analyses were performed using unpaired Student's $t$-tests, and analysis of variance was used for data subjected to multiple comparisons, followed by Bonferroni's post hoc analysis for comparisons between two groups. SPSS16.0 software (SPSS, Chicago, IL, USA) was used for all analyses. Values of $P<0.05$ were considered statistically significant.

\section{Results \\ Polyplex and liposome internalization mechanisms}

We studied the uptake of $\beta$-CD-(D3)7/MMP-9siRNA complexes and lip2000/MMP-9siRNA complexes using endocytosis inhibitors in HaCat cells. We labeled the MMP9siRNA with 5-FAM and assessed uptake by fluorescence microscopy and flow cytometry. Cell viability in the presence 
of different concentrations of endocytosis inhibitors was first determined to establish the optimal concentrations. Cellular toxicity was assessed using CCK- 8 assays. The results are shown in Figure 2. Both complexes exerted toxic effects to a certain extent on HaCat cells, as cell viability values dropped to $88 \%$ and $74 \%$ for polyplexes and liposomes, respectively. Amiloride alone showed limited toxicity toward $\mathrm{HaCat}$ cells after $5 \mathrm{~h}$ of incubation. The highest concentration tested $(100 \mu \mathrm{M})$ reduced viability by less than $10 \%$ (Figure 2A). Incubation in the presence of amiloride and polyplexes or liposomes reflected an almost additive effect in cell viability. Treatment with chlorpromazine concentrations higher than $10 \mu \mathrm{g} / \mathrm{mL}$ caused cytotoxicity (Figure 2B), while combined incubation with chlorpromazine $(15 \mu \mathrm{g} / \mathrm{mL})$ and polyplexes or liposomes resulted in $63.7 \%$ and $53.4 \%$ cell viability, respectively. After $5 \mathrm{~h}$ of incubation, HaCat cells were observed to be sensitive to genistein up to $200 \mu \mathrm{M}$ and to $\mathrm{M}-\beta-\mathrm{CD}$ up to $8 \mathrm{mM}$. On the other hand, higher concentrations led to $50 \%$ cell death (Figure $2 \mathrm{C}$ and $\mathrm{D}$ ) and were not used for further studies. On the basis of initial study results, we selected $100 \mu \mathrm{M}$ genistein, $50 \mu \mathrm{M}$ amiloride, $8 \mu \mathrm{g} / \mathrm{mL}$ chlorpromazine and $3 \mathrm{mM} \mathrm{M}-\beta-\mathrm{CD}$ for the subsequent experiments.

$\mathrm{HaCat}$ cells were preincubated for $1 \mathrm{~h}$ with $100 \mu \mathrm{M}$ genistein, $50 \mu \mathrm{M}$ amiloride, $8 \mu \mathrm{g} / \mathrm{mL}$ chlorpromazine, or $3 \mathrm{mM}$ M- $\beta-C D$. Next, the cells were incubated for $4 \mathrm{~h}$ with the 5-FAM-labeled polyplexes or liposomes in the presence of the corresponding inhibitor. Figure 3 shows that only $24 \%$ of the $\beta$-CD-(D3)7/MMP-9siRNA complexes were internalized into HaCat cells by the CDE and Cav-ME pathways; $70 \%$ of the lip2000/MMP-9siRNA complexes were absorbed by macropinocytosis and the Cav-ME pathway, while only $30 \%$ were absorbed by the CDE pathway into HaCat cells.

The internalization mode of complexes might affect the kinetics of their intracellular processing. The intracellular processing of polyplexes and liposomes internalized by different pathways into HaCat cells was unclear. In this study, we used laser scanning confocal microscopy to observe the location of complexes in the cytoplasm after intervention with
A

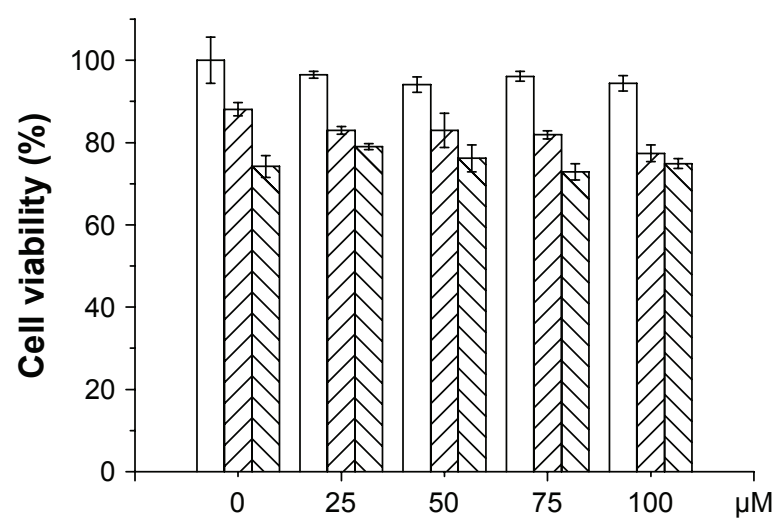

C

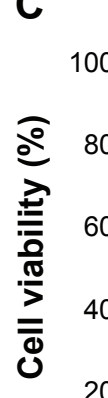

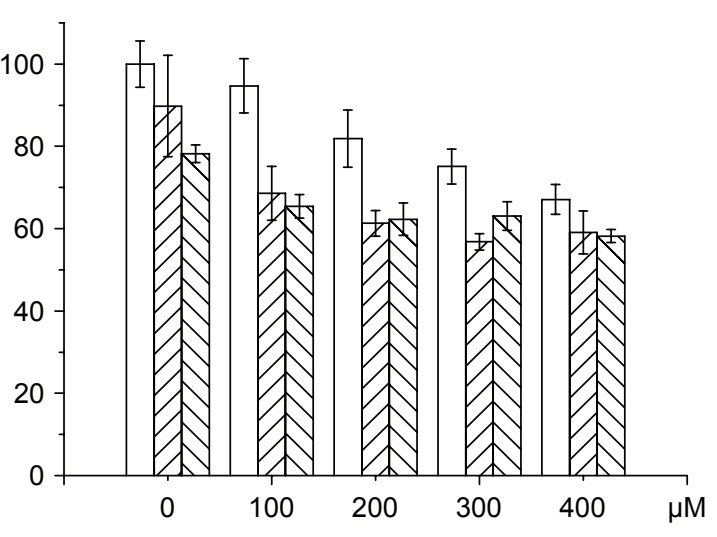

B

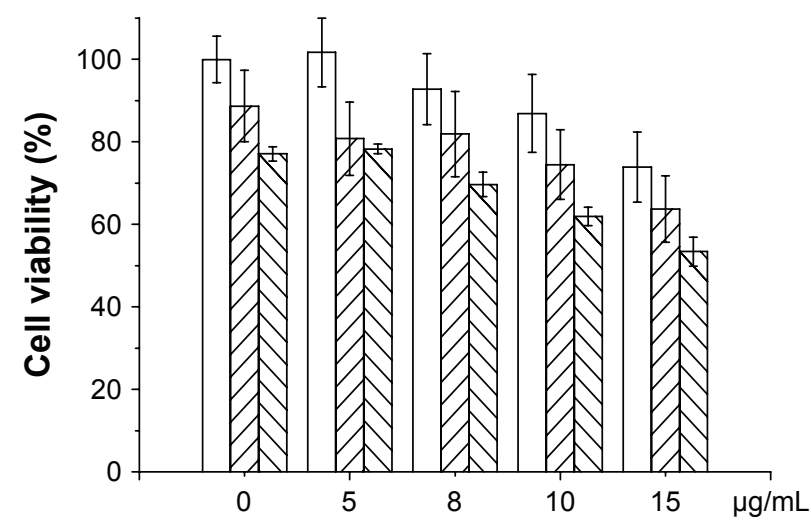

D

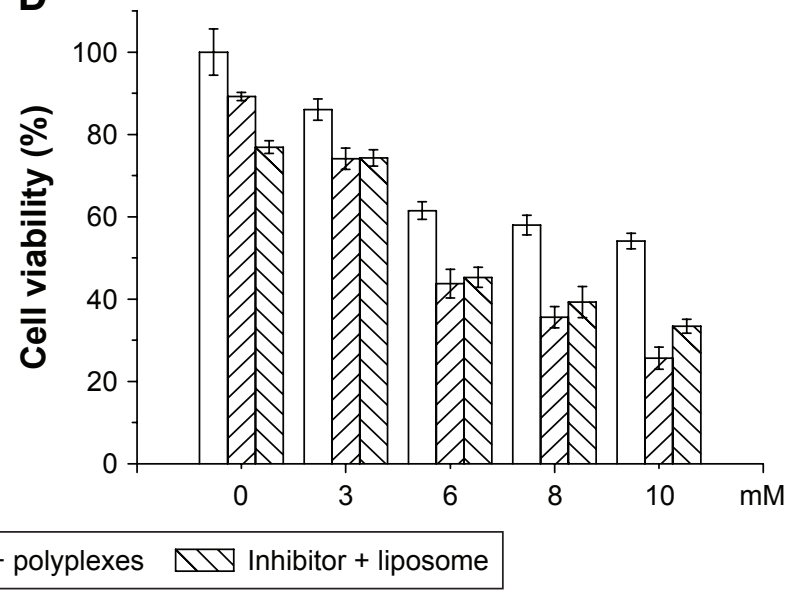

Figure 2 Cytotoxicity of different concentrations of endocytosis inhibitors to $\mathrm{HaCat}$ cells.

Notes: (A): amiloride, (B): chlorpromazine, (C): genistein, (D): M- $\beta-C D$. Data presented as mean \pm standard deviation. 


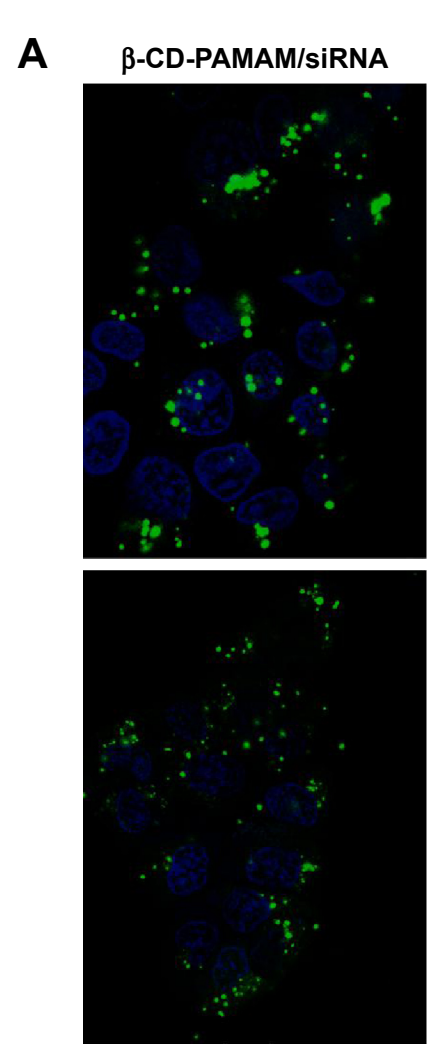

Genistein + $\beta$-CD-PAMAM/siRNA

lip2000/siRNA
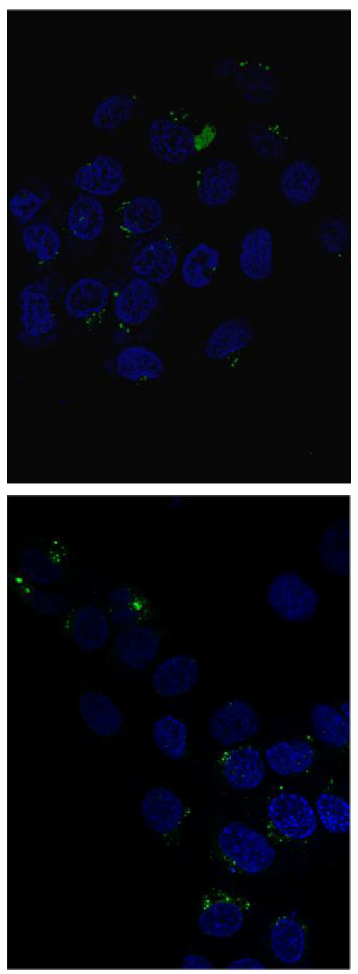

Genistein + lip2000/siRNA
Amiloride +

$\beta$-CD-PAMAM/siRNA
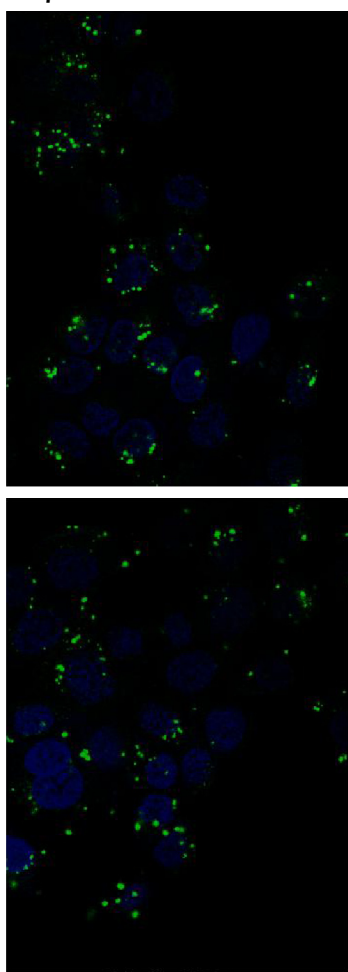

$M-\beta-C D+$

$\beta$-CD-PAMAM/siRNA

Amiloride + lip2000/siRNA
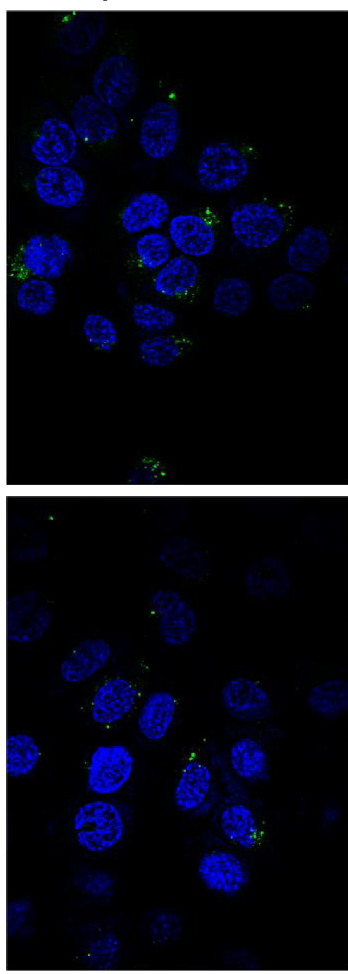

$M-\beta-C D+$ lip2000/siRNA
Chlorpromazine +

$\beta$-CD-PAMAM/siRNA

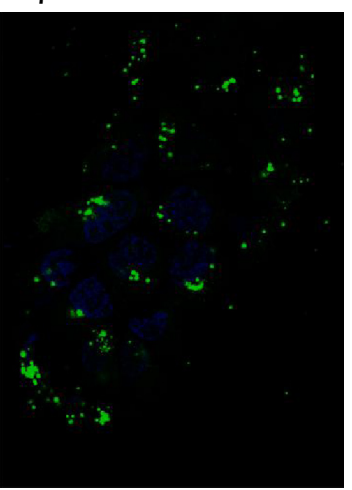

Chlorpromazine + lip2000/siRNA

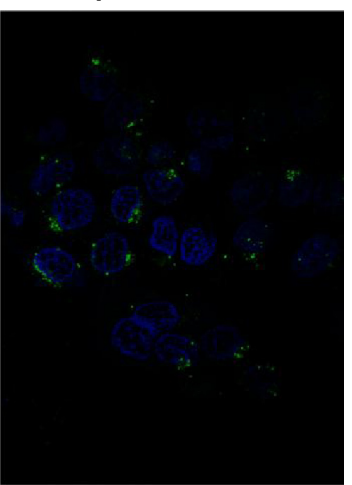



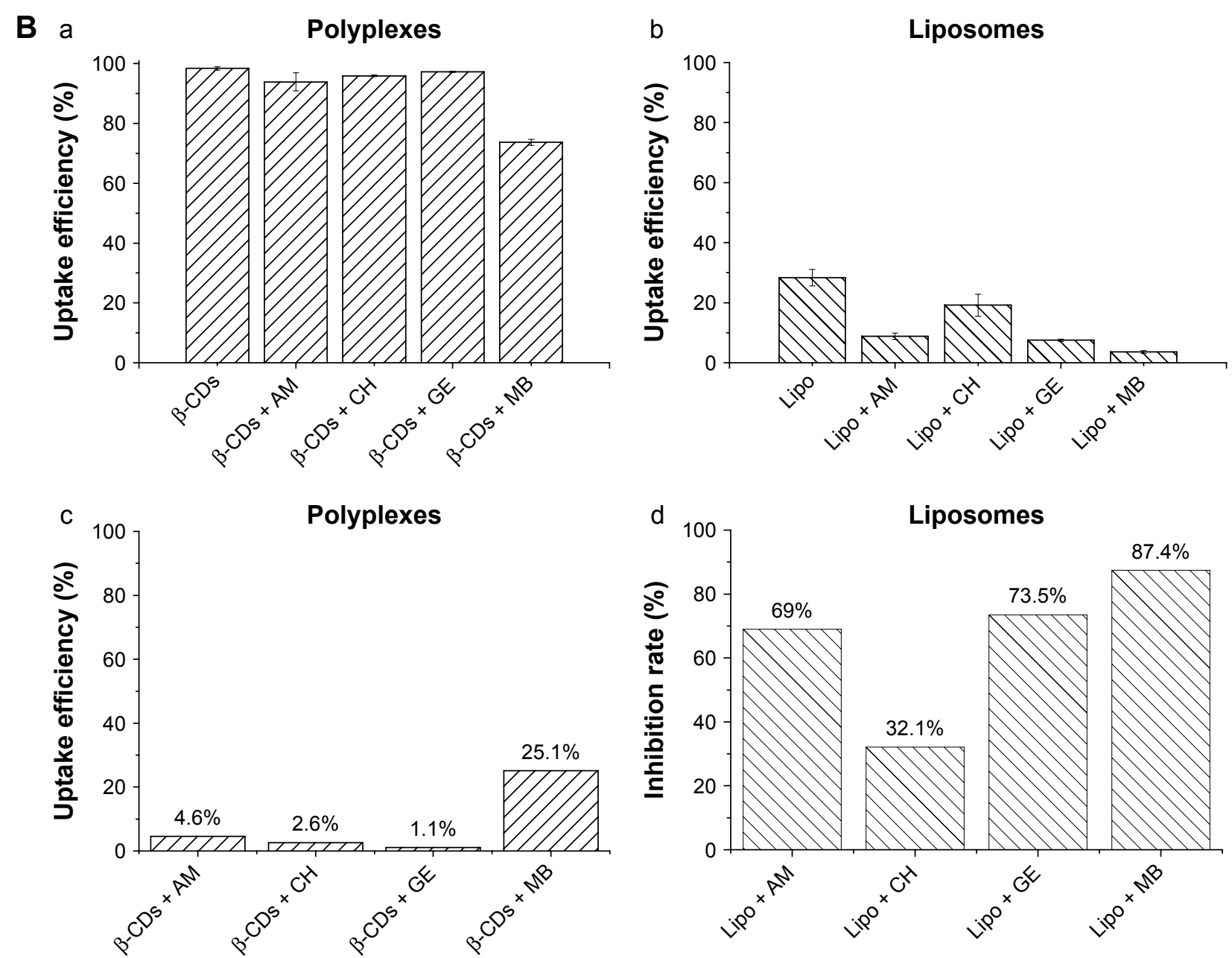

Figure 3 The uptake of $\beta$-CD-(D3)7/MMP-9siRNA complexes or lip2000/MMP-9siRNA complexes into HaCat cells after endocytosis inhibitors treatment.

Notes: (A): The internalization of 5-carboxyfluorescein (5-FAM)-loaded vesicles into HaCat cells observed by fluorescence microscopy. Punctuated green fluorescence was observed in $\beta$-CD-(D3)7/MMP-9siRNA complexes treatment groups. Less green fluorescence was observed in lip2000/MMP-9siRNA treatment group. (B): a) The uptake rates of $\beta-C D-(D 3) 7 / M M P-9 s i R N A$ complexes after different inhibitors treatment were quantified by flow cytometry; b) The uptake rates of lip2000/MMP-9siRNA complexes after different inhibitors treatment were quantified by flow cytometry; $c$ ) The uptake inhibition rates of $\beta$-CD-(D3)7/MMP-9siRNA complexes of each inhibition group compared with control group; d) The uptake inhibition rates of lip2000/MMP-9siRNA complexes of of each inhibition group compared with control group.

Abbreviations: $\beta-C D$ s, $\beta-C D-(D 3) 7 / s i R N A ;$ Lipo, lip2000/siRNA; AM, amiloride; $C H$, chlorpromazine; GE, genistein; MB, M- $\beta$-CD.

different endocytosis inhibitors. The colocalization of acidic organelle probes with polyplexes or liposomes indicated the combination of the complexes with the acidic organelles, which could by visualized as yellow particles. In the case of treatment with amiloride, chlorpromazine, or genistein, acidic organelles and polyplexes were partially colocalized, as indicated by few yellow particles in the cytoplasm (Figure 4). On the contrary, acidic organelles and polyplexes were not colocalized after treatment with $\mathrm{M}-\beta-\mathrm{CD}$, indicating that $\beta$-CD-(D3)7/MMP-9siRNA complexes internalized by nonclathrin- and non-caveolae-dependent endocytosis pathways would not be translocated into acidic organelles. It is worth noting that acidic organelle and liposome colocalization was observed in each group. Acidic organelles and liposomes were completely colocalized after treatment with chlorpromazine or M- $\beta-\mathrm{CD}$, while partial colocalization was observed after treatment with genistein or amiloride. The sd results suggested that the lip2000/MMP-9siRNA complexes internalized by the Cav-ME pathway and macropinocytosis would be translocated into acidic organelles.

We used RT-qPCR to measure the MMP-9 mRNA transcription level, resulting from treatment with the different endocytosis inhibitors, to explore the effect of endocytosis pathways on transfection efficiency. As shown in Figure 5, the levels of MMP-9 mRNA in the groups transfected with $\beta$-CD-(D3)7/MMP-9siRNA complexes were not upregulated, suggesting that no endocytosis inhibitor could completely suppress the interference effect of MMP-9siRNA. On the contrary, the levels of MMP-9 mRNA were all increased in the groups transfected with 

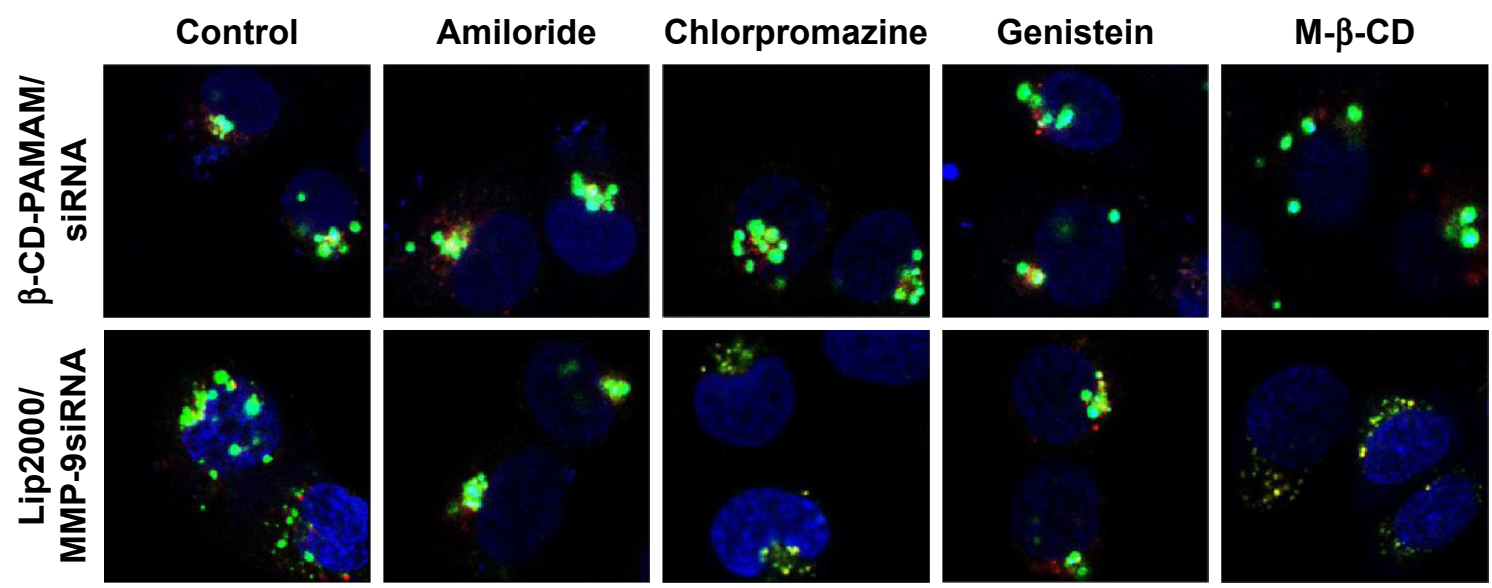

Figure 4 Colocalization of polyplexes or liposomes with acidic organelles after different internalization modes.

Notes: Acidic organelles: late endosomes and lysosomal; Green: 5-FAM labeled polyplexes or 5-FAM labeled liposomes; Red: LysoTracker DND-99 which is incorporated into liposome membrane; Blue: Hoechst 33342 which is the probe of nucleolus. Yellow: the colocalization of red acidic organelles probe with green fluorescent polyplexes or liposomes.

lip2000/MMP-9siRNA complexes, and MMP-9 expression was significantly upregulated in the chlorpromazine-treated group (with a relative value of $\sim 8$ ). These results indicated that the uptake of lip2000/MMP-9siRNA complexes by the CDE pathway could induce successful transfection.

\section{Intracellular polyplex and liposome processing}

The uptake of polyplexes and liposomes at different time points was also monitored by confocal microscopy using fluorescently labeled complexes and acidic organelle fluorescent probe. Images obtained at different time points are presented in Figure 6. Both the polyplexes and liposomes were partially combined with acidic organelles at $4 \mathrm{~h}$ after transfection, as indicated by the presence of many yellow particles, which tended to accumulate in the vicinity of the nucleus. At $12 \mathrm{~h}$, the polyplexes remained colocalized with acidic organelles, while the liposomes had separated from the acidic organelles. Fewer lip2000/MMP-9siRNA complexes were observed
A

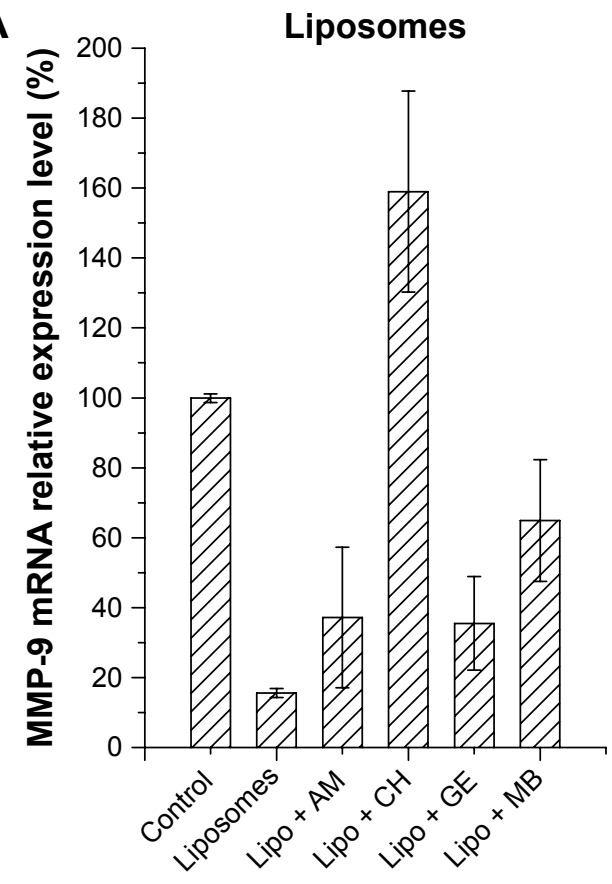

B

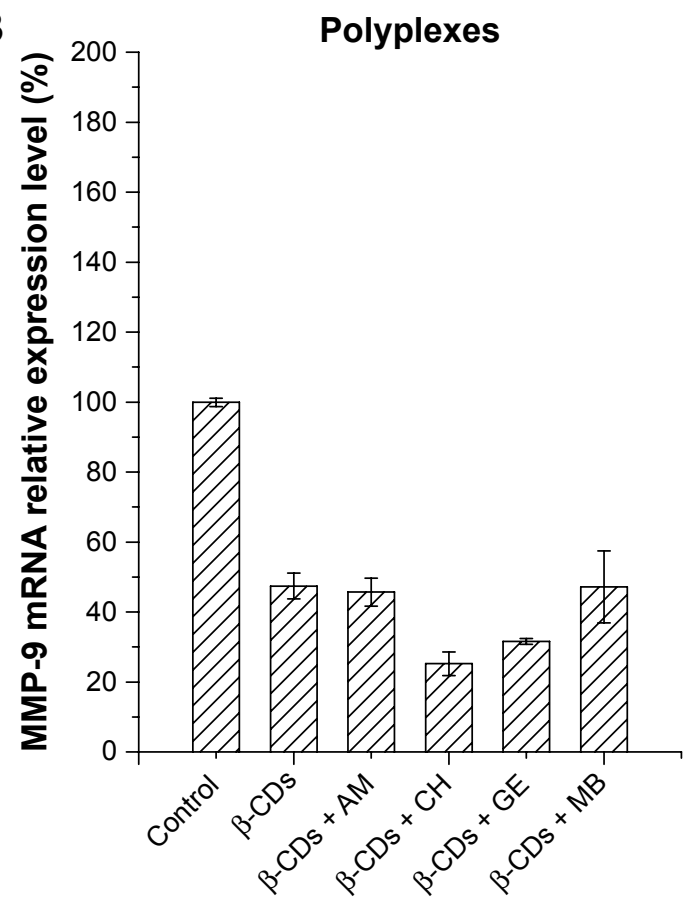

Figure 5 The RNA interference to MMP-9 messenger RNA induced by $\beta$-CD-(D3)7/MMP-9siRNA complexes or lip2000/MMP-9siRNA complexes that were internalized by different endocytosis pathways.

Notes: (A): lip2000/MMP-9siRNA complexes; (B): $\beta$-CD-(D3)7/MMP-9siRNA complexes.

Abbreviations: $\beta$-CDs, $\beta$-CD-(D3)7/siRNA; Lipo, lip2000/siRNA; AM, amiloride; $C H$, chlorpromazine; GE, genistein; MB, M- $\beta$-CD. 

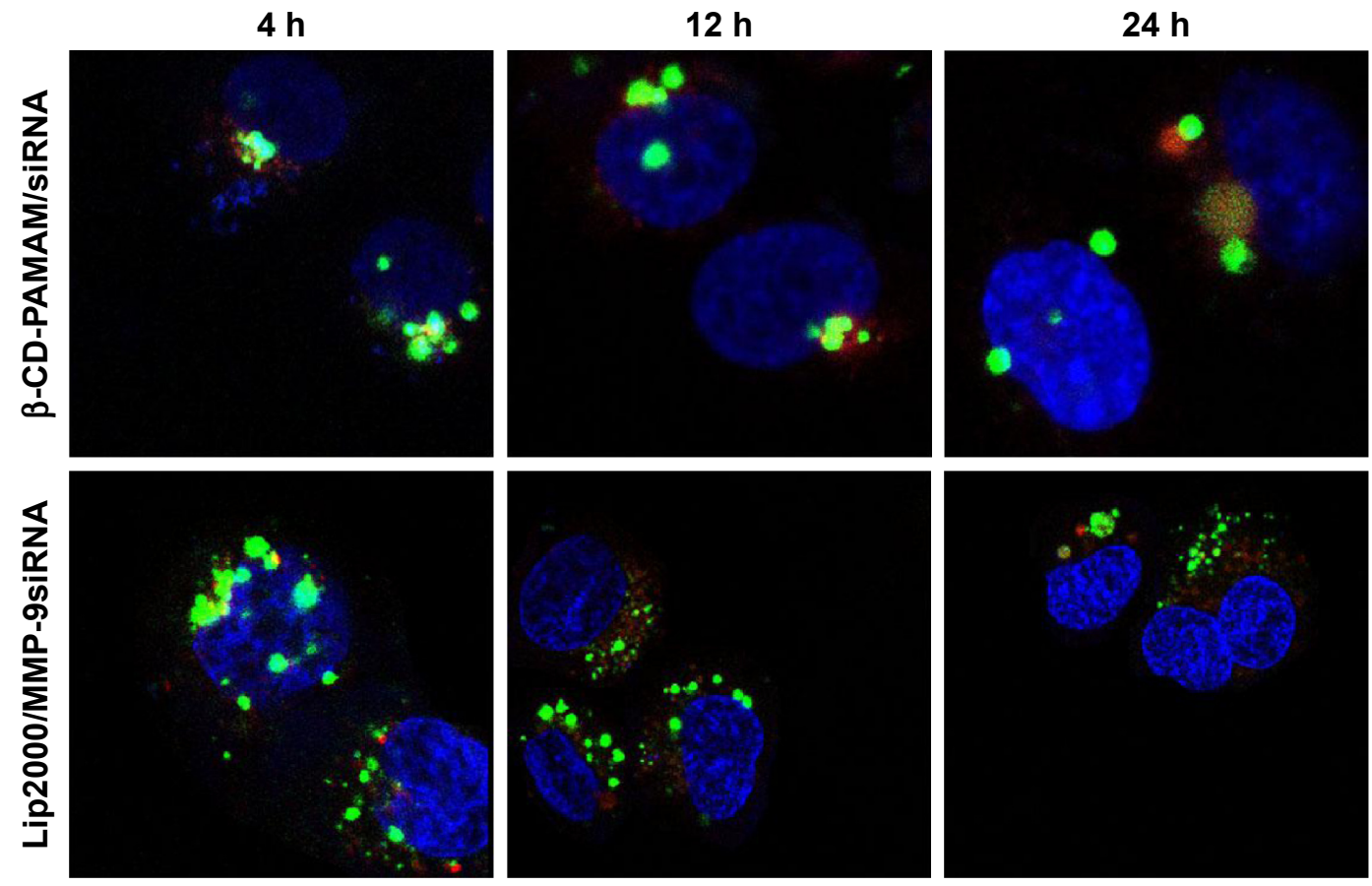

Figure 6 Uptake of polyplexes or liposomes was monitored by confocal microscopy.

Notes: Photographs were taken at 4, 12, and 24 h after transfection. Green: 5-FAM labeled polyplexes or liposomes; Red: LysoTracker DND-99; Blue: Hoechst 33342.

at $12 \mathrm{~h}$ than at $4 \mathrm{~h}$. As shown in Figure 6, the complete separation of polyplexes from acidic organelles was observed at $24 \mathrm{~h}$, and significantly fewer $\beta$-CD-(D3)7/ MMP-9siRNA complexes were observed at $24 \mathrm{~h}$ than at $4 \mathrm{~h}$.

\section{Endosomal escape mechanisms of polyplexes and liposomes}

As shown in Figure 6, we found that the liposomes separated from acidic organelles faster than did the polyplexes, and we aimed to determine whether this difference was related to the endosomal escape mechanism. We applied such endosomal acidification inhibitors as chloroquine and bafilomycin A1, and cell viability in the presence of different concentrations of the inhibitors was first determined to establish the optimal protocol for their use (Figure 7A). Finally, $50 \mu \mathrm{M}$ chloroquine and $50 \mathrm{nM}$ bafilomycin A1 were selected for the subsequent experiments.

We measured the effect of endosomal acidification on transfection efficiency by assaying the MMP-9 mRNA transcription level at $48 \mathrm{~h}$ after transfection (Figure 7B). In the case of chloroquine, no MMP-9 mRNA upregulation was observed in the group transfected with polyplexes or liposomes. However, in the case of bafilomycin A1, the MMP-9 mRNA levels were 8-fold and 7-fold greater than those in the polyplex and liposome positive control groups, respectively, suggesting that both complexes escaped from endolysosomes via the proton sponge mechanism.

\section{Discussion}

Our previous studies showed that $\beta$-CD-(D3)7/MMP9siRNA complexes had a higher internalization rate but a lower interference effect than lip2000/MMP-9siRNA complexes (uptake rate $98.4 \%$ vs $28.2 \%$, interference rate $44.2 \%$ vs $77.2 \%$ ). According to these results, we concluded that although $\beta$-CD-(D3)7/MMP-9siRNA complexes could be adequately absorbed, they could not effectively exert siRNA interference. This previous research confirmed that many barriers prevented gene vector-siRNA complexes from producing siRNA interference effects. Such barriers include being internalized by target cells, escaping from endolysosomes, and dissociating the siRNA cargo from the complex to achieve successful transfection. Hence we preliminarily explored these barriers in this study.

For the development of efficient vectors, it has to be taken into account that the internalization mechanism of the vehicles will affect the whole transfection process. The entry route will first influence the uptake quantitatively, while the intracellular fate among the different compartments within the cytoplasm (late endosomes, lysosomes, etc) will eventually outline the nuclear entry and the expression of the delivered genetic material. Endocytosis has been established as the main mechanism for the uptake of nonviral vectors into cells. ${ }^{4}$ Using selective endocytosis inhibitors was a method to explore the internalization mechanism of 


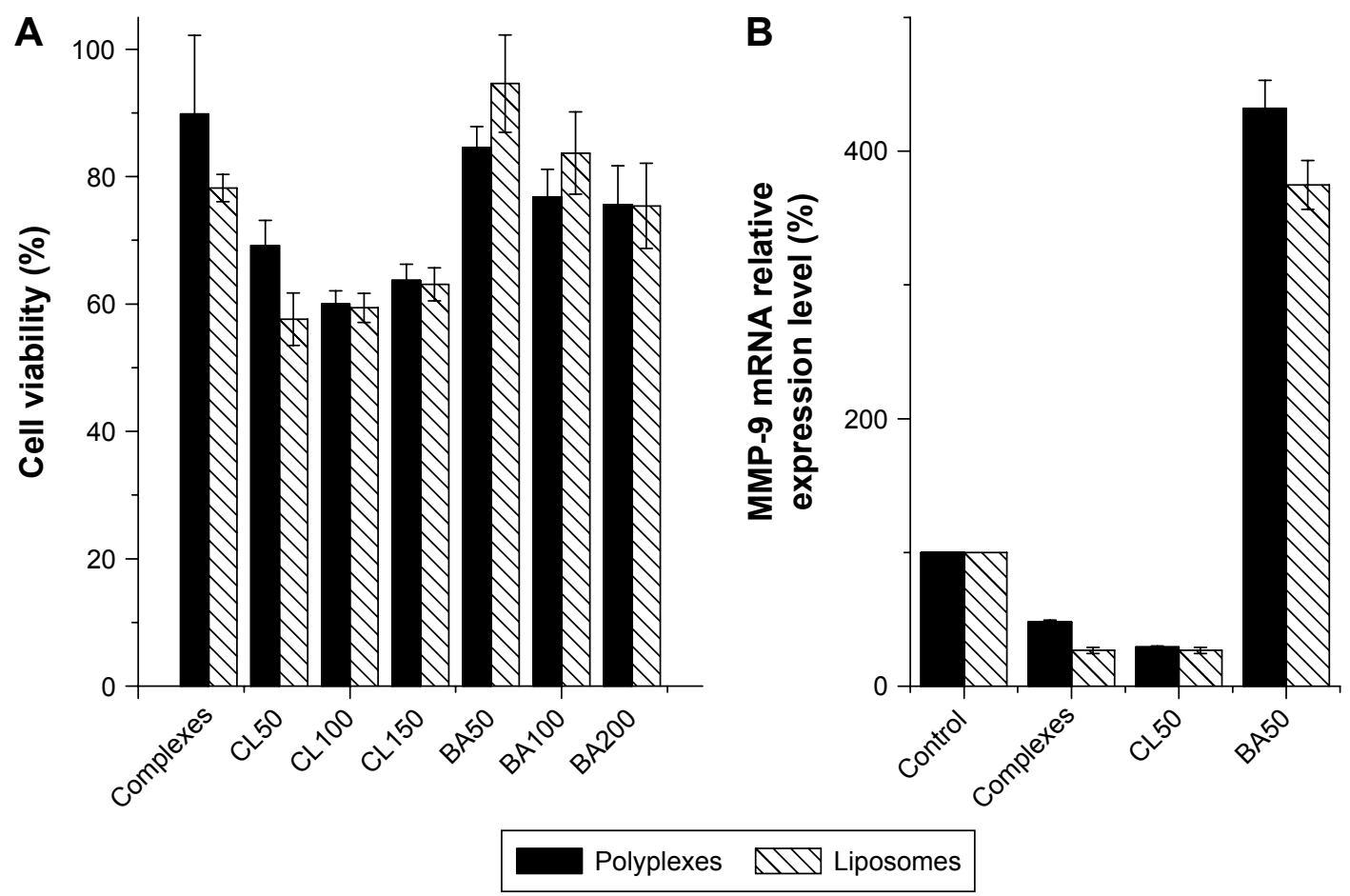

Figure 7 The effect of endosomal acidification to the transfection efficiency of $\beta$-CD-(D3)7/MMP-9siRNA complexes or lip2000/MMP-9siRNA.

Notes: (A): The HaCat cells viability after treating with different concentrations of endosomal acidification inhibitors. CL: chloroquine (concentration range: $50-150 \mu \mathrm{M}$ ); BA: Bafilomycin AI (concentration range: 50-200 nM). (B): The relative expression level of MMP-9 mRNA after different endosomal acidification inhibitors treatments quantified by RT-PCR.

Abbreviation: RT-PCR, real-time polymerase chain reaction.

nonviral gene vectors. Most studies in the field of particle uptake focus on pathways via the endocytosis of caveolae- or clathrin-coated vesicles and via macropinocytosis. We used the following four types of endocytosis inhibitors: amiloride (macropinocytosis), chlorpromazine (clathrin-mediated), genistein (caveolae-mediated), and $\mathrm{M}-\beta-\mathrm{CD}$ (caveolae- and clathrin-mediated). We studied the effects of the chemical endocytosis inhibitors on the uptake of polyplexes and liposomes by HaCat cells using fluorescence microscopy and flow cytometry. As shown in Figure 3, we concluded that the uptake of polyplexes might be largely mediated by another internalization mode, such as non-caveolae- and non-clathrinmediated pathways. However, the uptake of liposomes was diversified. Liposomes were mainly internalized by HaCat cells via macropinocytosis and the Cav-ME pathway and were marginally internalized by the clathrin-mediated endocytosis pathway.

Many factors regarding the characteristics of the carriers such as size, superficial charge, shape, or cell type can influence the internalization route. Size has been reported to be one of the most important influencing factors. Observations of the uptake of fluorescent polymers published by Rejman et $\mathrm{al}^{6}$ revealed that the size of particles determines the pathway of their entry into a cell as well as their subsequent intracellular trafficking. ${ }^{6,7}$ Cav-ME has been described as using vesicles that are usually small (50-80 $\mathrm{nm}$ in diameter), while the vesicles internalized in macropinocytosis, ie, macropinosomes, can have sizes of up to $5 \mu \mathrm{m}$. CDE is reportedly the preferred pathway for microspheres up to $200 \mathrm{~nm}$ in size. This was inconsistent with our results showing that $\beta$-CD(D3)7/MMP-9siRNA complexes $200 \mathrm{~nm}$ in diameter were not internalized by the above three endocytic pathways but by non-clathrin- and non-caveolae-mediated pathways instead. Although non-clathrin- and non-caveolae-mediated pathways are not currently well understood, a study by Lai et al also found that small polymeric particles entered live cells via a novel non-cholesterol-independent and non-clathrin- and non-caveolae-mediated mechanism, resulting in trafficking outside the endolysosomal pathway. ${ }^{8}$ Many viruses, such as the influenza, Sendai, and echoviruses, were also found to infect cells utilizing non-clathrin- and non-caveolaemediated pathways for transport to the golgi apparatus and, subsequently, to the endoplasmic reticulum. ${ }^{9,10}$

Previous research has reported that the mode of complex internalization might affect not only the intracellular processing kinetics but also the transfection efficiency. ${ }^{11}$ Thus, we studied the intracellular processing of polyplexes and liposomes internalized by different endocytosis pathways 
with acidic organelle fluorescent probes. The LysoTracker ${ }^{B}$ (Thermo Fisher Scientific, Waltham, MA, USA) probes are fluorescent probes with high selectivity for labeling and tracking acidic organelles in live cells. Polyplexes and liposomes were labeled with 5-FAM, and LysoTracker DND-99 labeling resulted in red fluorescence. We explored the colocalization of the two types of fluorescence using laser scanning confocal microscopy, which showed yellow particles in the cytoplasm. Through the analysis of images presented in Figure 4, we speculated that polyplexes absorbed by clathrin- or caveolae-mediated uptake pathways would be transported to acidic organelles such as lysosomes and secondary endosomes. Polyplexes internalized by macropinocytosis and non-clathrin- and non-caveolae-mediated pathways would avoid acidic organelles. Liposomes absorbed by macropinocytosis would be sent to acidic organelles, while liposomes internalized by clathrin-mediated endocytosis would avoid acidic organelles. Particles transported into acidic organelles might be digested, and lysosomal trafficking is routinely cited as a critical barrier to nonviral gene therapy. ${ }^{12}$ Lysosomal trafficking, characterized by a low $\mathrm{pH}$ and an environment rich in acidic hydrolases, poses particularly severe constraints on the efficient intracellular delivery of various chemical and biological molecules. A broad range of therapeutics, including DNA, RNA, and many proteins and chemotherapeutic agents, rapidly degrade in acidic environments. The trafficking of drug and gene carriers in acidic vesicles is a critical bottleneck in therapeutic delivery applications.

We also measured the effect of internalization mode on transfection efficiency by assaying the MMP-9 mRNA transcription level at $48 \mathrm{~h}$ after transfection (Figure 5). We concluded that polyplexes internalized by these three endocytosis pathways would not induce successful transfection. However, liposomes internalized by each type of endocytosis pathway, especially clathrin-mediated endocytosis pathways, might induce successful transfection.

As shown in Figure 4, we found that both $\beta$-CD-(D3)7/ MMP-9siRNA complexes and lip2000/MMP-9siRNA complexes could partially be transported into acidic organelles, resulting in either digestion or escape into the cytoplasm. Therefore, we loosely compared the digestion rate and endosomal escape rate of polyplexes and liposomes by confocal microscopy using fluorescently labeled complexes and fluorescent acidic organelle probes. As shown in Figure 6, we captured images at 4, 12, and $24 \mathrm{~h}$ after transfection. Through the analysis of images presented in Figure 6, we proposed that the lip2000/MMP-9siRNA complexes could escape from acidic organelles more quickly than the $\beta$-CD-(D3)7/MMP-9siRNA complexes. Digestion by acidic organelles affected the lip2000/MMP-9siRNA complexes less than the $\beta$-CD-(D3)7/MMP-9siRNA complexes, which may be the main reason for the lower transfection efficiency of the $\beta$-CD-(D3)7/MMP-9siRNA complexes.

As the polyplexes and liposomes showed different endosomal escape rates, we explored the possible endosomal escape mechanisms of the polyplexes and liposomes. The "proton sponge effect" was proposed as one of the potential mechanisms for the endosomal release of the polymers. ${ }^{13}$ The ATPase proton pump inhibitor bafilomycin A1 and the lysosomotropic base chloroquine were thus applied in our study, both of which would disturb endosomal acidification and increase the endosomal $\mathrm{pH}$. We measured the effect of the endosomal acidification inhibitors on transfection efficiency by assaying the MMP-9 mRNA transcription level at $48 \mathrm{~h}$ after transfection to explore the endosomal release mechanisms (Figure 7B). The results showed that while bafilomycin A1 could inhibit the endosomal escape of complexes, thus downregulating the siRNA interference effect, chloroquine failed to inhibit the endosomal escape of complexes. These results could be attributed to the concentration used in the current study $(50 \mu \mathrm{M})$, as chloroquine has been shown to raise endosomal $\mathrm{pH}$ levels at concentrations over 0.6 M. However, it is possible that HaCat cells are more sensitive to chloroquine treatment. We observed substantial cytotoxicity in $\mathrm{HaCat}$ cells treated with chloroquine concentrations greater than $50 \mu \mathrm{M}$, which precluded further endosomal acidification to achieve the endosomal escape of polyplexes and liposomes in the current study. We concluded that both polyplexes and liposomes were dependent on the proton sponge mechanism for endosomal escape. Ouyang et al, described the mode of siRNA release from cationic polymers as the proton sponge mechanism, ${ }^{13}$ which implies that polymers sequester the protons transported into the endosomal compartment, thus preventing endosomal acidification. Subsequently, the increased osmolarity in this compartment causes the influx of water, which eventually leads to endosome lysis and the release of its contents into the cytoplasm.

In brief, we could increase the transfection efficiency of $\beta$-CD-(D3)7 by transforming the vector's structure to facilitate the interaction with the epidermal cells surface, and thus, improve the internalization by non-clathrin- and non-caveolae-dependent endocytosis pathways, and then increase the release from the endosomal compound to get successful transfection. We will apply the transformed 
$\beta$-CD-(D3)7vector/MMP-9siRNA complexes into diabetic foot skin to promote wound healing in the clinic. Our group is working on related research.

\section{Conclusion}

$\beta$-CD-(D3)7/MMP-9siRNA complexes were internalized by non-caveolae- and non-clathrin-mediated pathways and could induce successful transfection, and lip2000/MMP-9siRNA complexes internalized by CDE could induce successful transfection. Although both of these complexes escaped from endolysosomes via the proton sponge mechanism, we still found that the lip2000/MMP-9siRNA complexes escaped more quickly from the acidic organelles than did the $\beta$-CD(D3)7/MMP-9siRNA complexes and that digestion by acidic organelles affected the lip2000/MMP-9siRNA complexes less than the $\beta$-CD-(D3)7/MMP-9siRNA complexes. This effect may be the reason for the lower transfection efficiency of $\beta$-CD-(D3)7. Our study provided insights for further improvements in the molecular design of the polycationic amphiphilic cyclodextrin vector, eg, the incorporation of functional elements promoting endo-lysosomal escape and restraining digestion by acidic organelles. Research in this direction is currently underway in our laboratories.

\section{Acknowledgments}

This study was supported by the National Natural Science Foundation of China (81500640, 81471034, 81270916, $81370910,81670764)$, the Major Projects of People's Livelihood Science and Technology in Guangzhou (201300000102); Applied Science and Technology Research and Development in Guangdong Province (2016B020238001), and the National High Technology Research and Development Program (2015AA020927). This work was also supported by the Key Laboratory of Malignant Tumor Molecular Mechanisms and Translational Medicine of the Guangzhou Bureau of Science and Information Technology and the Key Laboratory of
Malignant Tumor Gene Regulation and Target Therapy of Guangdong Higher Education Institutes.

\section{Disclosure}

The authors report no conflicts of interest in this work.

\section{References}

1. Li N, Luo HC, Yang C, et al. Cationic star-shaped polymer as an siRNA carrier for reducing MMP-9 expression in skin fibroblast cells and promoting wound healing in diabetic rats. Int J Nanomedicine. 2014;9: 3377-3387.

2. Schott JW, Morgan M, Galla M, Schambach A. Viral and synthetic RNA vector technologies and applications. Mol Ther. 2016;24(9):1513-1527.

3. Namvar A, Bolhassani A, Khairkhah N, Motevalli F. Physicochemical properties of polymers: an important system to overcome the cell barriers in gene transfection. Biopolymers. 2015;103(7):363-375.

4. Perez Ruiz de Garibay A. Endocytosis in gene therapy with non-viral vectors. Wien Med Wochenschr. 2016;166(7-8):227-235.

5. Khalil IA, Kogure K, Akita H, Harashima H. Uptake pathways and subsequent intracellular trafficking in nonviral gene delivery. Pharmacol Rev. 2006;58(1):32-45.

6. Rejman J, Oberle V, Zuhorn IS, Hoekstra D. Size-dependent internalization of particles via the pathways of clathrin- and caveolae-mediated endocytosis. Biochem J. 2004;377(1):159-169.

7. Parakhonskiy B, Zyuzin MV, Yashchenok A, et al. The influence of the size and aspect ratio of anisotropic, porous $\mathrm{CaCO}_{3}$ partcles on their uptake by cells. J Nanobiotechnology. 2015;13:53.

8. Lai SK, Hida K, Man ST, et al. Privileged delivery of polymer nanoparticles to the perinuclear region of live cells via a non-clathrin, nondegradative pathway. Biomaterials. 2007;28(18):2876-2884.

9. Gerondopoulos A, Jackson T, Monaghan P, Doyle N, Roberts LO. Murine norovirus-1 cell entry is mediated through a non-clathrin-, noncaveolae-, dynamin- and cholesterol-dependent pathway. J Gen Virol. 2010;91(6):1428-1438.

10. Sieczkarski SB, Whittaker GR. Dissecting virus entry via endocytosis. $J$ Gen Virol. 2002;83(7):1535-1545.

11. Hwang ME, Keswani RK, Pack DW. Dependence of PEI and PAMAM gene delivery on clathrin- and caveolin-dependent trafficking pathways. Pharm Res. 2015;32(6):2051-2059.

12. Cardarelli F, Digiacomo L, Marchini C, et al. The intracellular trafficking mechanism of Lipofectamine-based transfection reagents and its implication forgene delivery. Sci Rep. 2016;11(6):25879.

13. Ouyang D, Zhang H, Parekh HS, Smith SC. The effect of pH on PAMAM dendrimer-siRNA complexation: endosomal considerations as determined by molecular dynamics simulation. Biophys Chem. 2011; 158(2-3):126-133.
International Journal of Nanomedicine

\section{Publish your work in this journal}

The International Journal of Nanomedicine is an international, peerreviewed journal focusing on the application of nanotechnology in diagnostics, therapeutics, and drug delivery systems throughout the biomedical field. This journal is indexed on PubMed Central, MedLine, CAS, SciSearch ${ }^{\circledR}$, Current Contents $® /$ Clinical Medicine,

\section{Dovepress}

Journal Citation Reports/Science Edition, EMBase, Scopus and the Elsevier Bibliographic databases. The manuscript management system is completely online and includes a very quick and fair peer-review system, which is all easy to use. Visit http://www.dovepress.com/ testimonials.php to read real quotes from published authors. 